\title{
DISCOVERY AND STRUCTURE-ACTIVITY RELATIONSHIP OF POTENT AND SELECTIVE COVALENT INHIBITORS OF SMYD3
}

\author{
E.V. Manasova', V.A. Vasilichin', A.A. Shtil', A. Altieri', M.D. Parenti' ${ }^{2}$, \\ C. Ferroni' ${ }^{2}$, G. Varchi², A. Del Rio' ${ }^{2}$ and A.V. Kurkin' ${ }^{1}$ \\ ${ }^{1}$ Lomonosov Moscow State University, Department of Chemistry, Moscow, Russia. \\ ${ }^{2}$ Institute of Organic Synthesis and Photoreactivity - ISOF, \\ Italian National Research Council, Via Gobetti 101, 40129 Bologna, Italy.
}

DOI: 10.19163/MedChemRussia2021-2021-230

E-mail:kurkin@direction.chem.msu.ru

SMYD3, a member of the SMYD lysine methylase family, plays an important role in methylation of histone and non-histone targets. An aberrant SMYD3 expression contributes to carcinogenesis; SMYD3 up-regulation has been proposed a prognostic marker in various solid cancers. The potential of SMYD3 as a drug target for cancer treatment suggests that well-designed covalent SMYD3 inhibitors could serve not only to design future drugs but also to validate the role of SMYD3 as an oncotarget. In this study we report the first generation of 4-aminopiperidine-based covalent SMYD3 inhibitors that were synthetically modified with an appropriate reactive cysteine trap to covalently bind CYS186. The resulting covalent SMYD3 inhibitors can act with a higher potency and selectivity in respect to reversible SMYD3 inhibitors and can exert long-lasting effects that will translate in high antiproliferative properties. The synthesis of piperidine-based compounds bearing the reactive moiety at the 4-position of the piperidine scaffold is reported in the Scheme:

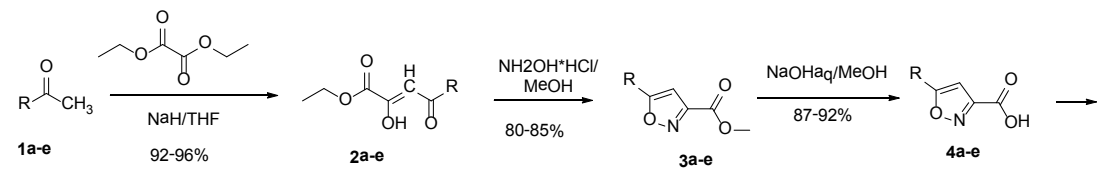

$\mathrm{R}=\mathrm{CH}_{3}, \mathrm{Ph}$, cyclopropyl, 4-CH $\mathrm{CH}_{3} \mathrm{Ph}$-propyl

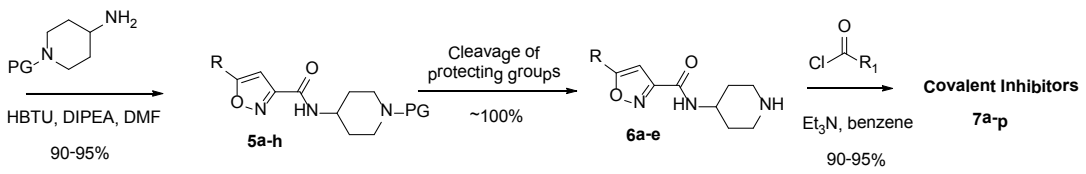

$\mathrm{PG}=\mathrm{Boc}, \mathrm{Alloc}, \mathrm{Ac}$

While other small molecular weight SMYD3 inhibitors have been reported, and a similar isoxazole analog of this series has been exemplified ${ }^{1,2}$ the compounds disclosed here represent a detailed SAR study of this class. In particular, the close analog EPZO28862 showed excellent inhibition of SMYD3. So, the compounds disclosed in this work represent a novel structural class of SMYD3 inhibitors. Modification of HTS hit, compound 7a, with the structure guided SAR study led to compound $7 \mathbf{p}$, a potent, selective, orally bioavailable small molecule SMYD3 inhibitor.

The reported study was funded by RFBR and CNR, project number 20-53-7808.

\section{References}

1. Mitchell, L. H. et al., ACS Med. Chem. Lett. 2016, 7 (2), 134-8.

2. Thomenius, M. J. et al., PLoS One 2018, 13 (6), No. e0197372. 